\title{
T-DNA site identification by Cas9-targeted and whole-genome Nanopore sequencing of Arabidopsis thaliana
}

\author{
Gvaramiya S. ${ }^{1 *}$, Merkulov P. ${ }^{1}$, Omarov M. ${ }^{1,2}$, Komakhin R. ${ }^{1}$, Kirov I. ${ }^{1}$ \\ ${ }^{1}$ All-Russia Research Institute of Agricultural Biotechnology, Moscow, Russia \\ ${ }^{2}$ National Research University Higher School of Economics, Moscow, Russia \\ * email: sofia.gvaramia@gmail.com
}

The identification of T-DNA insertion sites after a transformation experiment is timeconsuming and a challenging task. Traditional methods require several generations of selfing and segregation analysis followed by whole-genome sequencing. Without this information, the impact of T-DNA on phenotype can be misinterpreted. To deal with this problem, we optimized Cas9-targeted Nanopore sequencing of T-DNA insertion sites and compared this approach with the whole-genome long-read sequencing. For this, we generated transgenic $A$. thaliana plants with overexpression of GAG protein - the main component of assembling retrotransposon`s capsid. F2 plants were obtained and segregation analysis was carried out to select plant material with a single T-DNA insertion locus. With the use of CRISPR-P 2.0, we designed sgRNA for T-DNA. Synthesized sgRNAs were used for ribonucleoproteins assembly and Nanopore library preparation. The obtained reads were analysed by a newly developed pipeline and $\mathrm{T}$ DNA insertion sites were successfully determined. To verify our data, we perform whole-genome sequencing of 4 independent transgenic lines using native barcodes (EXP-NBD-104) and Ligation Sequencing Kit (SQK-LSK-109) on MinION (Oxford Nanopore Technologies, England). Approximately 400,000 reads were obtained from R10.3 flow cell with an estimated N50 23,4 kb and 10-18x coverage. With our specific bioinformatic pipeline, designed to define the insertion sites, we observed homozygous and heterozygous insertions. In addition, a plant with multiple integration events resulted in a single tandemly organized T-DNA insertion was identified. PCR was performed to confirm all T-DNA insertions. The advantages and features of Nanopore sequencing for T-DNA integration site identification will be discussed. The obtained results are useful for selection of suitable transgenic plants to study the effect of GAG protein overexpression on genome and transcriptome constitution.

Acknowledgements: The reported study was funded by RFBR, project number 20-3470032. 